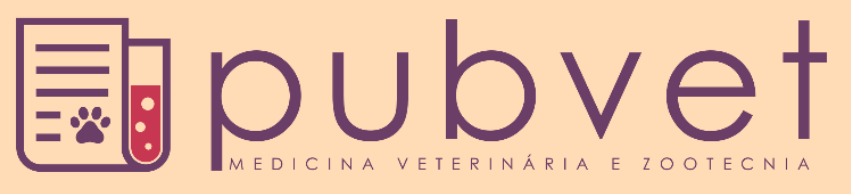

https://doi.org/10.31533/pubvet.v15n01a732.1-8

\title{
Comportamento e desempenho reprodutivo de marrãs mantidas em diferentes sistemas de criação
}

\author{
Rodrigo Fortunato de Oliveira ${ }^{1 *} \bullet$ (D), Rita da Trindade Ribeiro Nobre Soares ${ }^{1} \bullet$, Rayanne \\ Prates de Andrade ${ }^{2} \bullet$, Leonardo Demier $\operatorname{Cardoso}^{1 \oplus}$, Edison Torres da Silva Júnior $^{1 \oplus}$ \\ ${ }^{1}$ Universidade Estadual do Norte Fluminense Darcy Ribeiro, Centro de Ciências e Tecnologias Agropecuárias, Laboratório de Zootecnia e \\ Nutrição Animal. Campos dos Goytacazes - RJ Brasil. \\ ${ }^{2}$ Médica Veterinária, Universidade Iguaçu. Itaperuna - RJ Brasil. \\ *Autor para correspondência, E-mail: fortunatorodrigo@ymail.com
}

Resumo. A busca pela melhoria da qualidade de vida vem sendo estudada pela utilização de manejos e instalações que possam melhorar o bem-estar dos animais de produção. $\mathrm{O}$ Objetivo deste trabalho foi avaliar a influência de diferentes sistemas de alojamento durante as fases de gestação e maternidade sobre o comportamento e desempenho de marrãs. Os animais foram distribuídos em um delineamento inteiramente casualizado, com dois tratamentos e quatro repetições, sendo as marrãs consideradas a unidade experimental. Os tratamentos foram: Grupo 1 - Sistema de confinamento em gaiolas; Grupo 2 - Sistema de criação em piquete e baia. Foram coletados dados de comportamento e desempenho reprodutivo das marrãs e o desempenho produtivo dos leitões. As marrãs na fase de gestação alojadas nas gaiolas de confinamento, permaneceram na maior parte do tempo inativas nos três horários de coletas (8:00 - 10:00, 12:00 - 14:00 e 16:00 - 18:00) e menor tempo em alerta entre os horários 8:00 - 10:00 e 12:00 - 14:00, comparado as marrãs criadas em piquete. Os animais alojados em piquetes permaneceram em alerta 87,3\% acima do tempo de observação comparado as fêmeas alojadas em gaiolas individuais. As fêmeas criadas em piquetes apresentaram maior $(\mathrm{P}<0,05)$ número de leitões nascidos vivos. Todos os índices avaliados nos leitões não apresentaram diferenças $(\mathrm{P}<0,05)$ entre os sistemas de alojamento. $\mathrm{O}$ sistema de piquetes na gestação proporcionou uma melhora do bem-estar das marrãs, mas não foi o suficiente para melhorar o desempenho reprodutivo e dos leitões na maternidade.

Palavras-chave: Bem-estar animal, comportamento, gaiolas de gestação, piquetes

\section{Behavior and gilts reproductive performance kept in different breeding systems}

Abstract. The search for improving the quality of life has been studied through the use of
management and facilities that can improve the welfare of farm animals. The objective of
this work was to evaluate the influence of different housing systems during the gestation
and maternity phases on the behavior and performance of gilts. The animals were
distributed in a completely randomized design, with two treatments and four replications,
with the gilt being considered the experimental unit. The treatments were: Group 1 - Caged
confinement system; Group 2 - Picket and stall breeding system. Data on behavior and
reproductive performance of gilts and productive performance of piglets were collected.
The gilts in the gestation phase housed in the confinement cages, remained mostly inactive
at the three collection times $(8: 00-10: 00,12: 00-14: 00$ and 16:00 - 18:00) and shorter
alert time between 8:00 - 10:00 and 12:00 - 14:00, compared to gilts reared in paddocks.
Animals housed in paddocks remained on alert $87.3 \%$ above the observation time 
compared to females housed in individual cages. Females raised in paddocks had a higher $(\mathrm{P}<0.05)$ number of piglets born alive. All indexes evaluated in piglets did not show differences $(\mathrm{P}>0.05)$ between housing systems. The paddock system during pregnancy provided an improvement in the welfare of the gilts, but it was not enough to improve the reproductive and piglet performance in the maternity.

Keywords: Animal welfare, behavior, gestation cages, paddocks

\section{Comportamiento y rendimiento reproductivo de las cerdas mantenidas en diferentes sistemas de cría}

Resumen. La búsqueda de mejorar la calidad de vida se ha estudiado mediante el uso de la
gestión y las instalaciones que pueden mejorar el bienestar de los animales de granja. El
objetivo de este trabajo fue evaluar la influencia de los diferentes sistemas de producción
durante las fases de gestación y maternidad en el comportamiento y el rendimiento de las
primerizas. Los animales se distribuyeron en un diseño completamente al azar, con dos
tratamientos y cuatro repeticiones, considerándose las primerizas como la unidad
experimental. Los tratamientos fueron: Grupo 1 - Sistema de confinamiento enjaulado;
Grupo 2 - Sistema de cría de piquetes y corrales. Se recopilaron datos sobre el
comportamiento y el rendimiento reproductivo de las primerizas y el rendimiento
productivo de los lechones. Las primerizas en la fase de gestación alojadas en las jaulas de
confinamiento, permanecieron mayormente inactivas en los tres horarios de recolección
(08:00 - 10:00, 12:00 - 14:00 y 16:00 - 18:00) menor tiempo de alerta entre las $08: 00$ -
10:00 y las 12:00 - 14:00, en comparación con las primerizas criadas en potreros. Los
animales alojados en potreros permanecieron en alerta $87,3 \%$ por encima del tiempo de
observación en comparación con las hembras alojadas en jaulas individuales. Las hembras
criadas en potreros tenían un mayor número (P < 0.05$)$ de lechones nacidos vivos. Todos
los índices evaluados en lechones no mostraron diferencias $(\mathrm{P}>0,05)$ entre los sistemas de
alojamiento. El sistema de piquetes durante la preñez proporcionó una mejora en el
bienestar de las primerizas, pero no fue suficiente para mejorar el rendimiento reproductivo
y de los lechones en la maternidad.

Palabras clave: Bienestar animal, comportamiento, jaulas de gestación, potreros

\section{Introdução}

No cenário mundial, o Brasil se destaca ocupando o quarto lugar como maior produtor e exportador de suínos, com 3,5\% da produção, $16 \%$ das exportações e crescente inserção internacional (ANUALPEC, 2020; FAPRI, 2020).

O sistema de criação confinado foi criado com o intuito de reduzir o trabalho e a perda energética dos animais, ganhar espaço e melhorar o controle ambiental (Nazareno et al., 2012). No entanto, os problemas de bem-estar animal são agravados (Oliva et al., 2014), uma vez que a condição imposta restringe o comportamento natural dos animais (Nazareno et al., 2012).

Para melhor produtividade da fêmea suína, o ideal seria a escolha de um sistema de produção que fornece, além de cuidados especiais, o bem-estar animal. Na produção intensiva, os animais são criados confinados em baias ou gaiolas, num espaço relativamente pequeno, apresentando preocupação com a produtividade e economicidade do sistema. O sistema de criação de suínos ao ar livre apresenta diversas vantagens em relação ao sistema confinado, como baixo investimento inicial (Sousa et al., 2012), menor produção de odores indesejáveis, melhores condições ambientais e melhoria nas condições de bem-estar dos animais (Veloni et al., 2013; Kongsted \& Sørensen, 2017).

As granjas de suínos que produzem leitões no Brasil utilizam o modelo de produção com gaiolas individuais. As fêmeas passam cerca de $80 \%$ da vida num espaço médio de $63 \mathrm{~cm}$ de largura por $2,2 \mathrm{~m}$ de comprimento e $1,05 \mathrm{~m}$ de altura. As gaiolas individuais podem gerar problemas musculares que, com o tempo, tornam difícil até mesmo os atos de deitar e levantar. O estresse do confinamento também leva 
o animal a comportamentos não naturais como morder repetidamente as barras de ferro das gaiolas (Budiño et al, 2014).

Desta forma, o objetivo deste trabalho foi avaliar o comportamento e desempenho reprodutivo de marrãs bem como o desempenho dos respectivos leitões em diferentes sistemas de alojamento durante a gestação.

\section{Material e métodos}

O uso de animais para essa pesquisa foi aprovado pela CEUA (Comissão de Ética para a Utilização de Animais) com o número de registro 080/12. A presente pesquisa foi conduzida no Setor de Suinocultura do Laboratório de Zootecnia e Nutrição Animal (LZNA), do Centro de Ciências e Tecnologias Agropecuárias (CCTA), situado no Colégio Agrícola Antônio Sarlo, no município de Campos dos Goytacazes, Rio de Janeiro. A cidade de Campos dos Goytacazes situa-se a uma Latitude de $21^{\circ} 45^{\prime} 15^{\prime \prime}$ e Longitude de $41^{\circ} 19^{\prime} 28^{\prime}$ ', com Altitude de 13 metros. O município de Campos dos Goytacazes situa-se na região Norte Fluminense do estado do Rio de Janeiro. Segundo a classificação climática de Köppen \& Geiger (1928), a região Norte Fluminense insere-se na classe Aw, isto é, tropical úmido, com verão chuvoso, inverno seco e temperatura do mês mais frio superior a $18^{\circ} \mathrm{C}$. A temperatura média anual situa-se em torno de $24^{\circ} \mathrm{C}$, sendo a amplitude térmica pequena e a normal climatológica da precipitação pluviométrica igual a 1.055,3 mm (Ramos et al., 2009).

Foram utilizadas oito marrãs da linhagem Fertilis 20 (Genétiporc), com peso médio de 120,85 \pm 0,7 $\mathrm{kg}$. As matrizes foram distribuídas em delineamento inteiramente casualizados, com dois grupos e quatro repetições. As marrãs foram avaliadas por um ciclo reprodutivo, contando gestação, lactação e período para retorno ao cio.

Os grupos avaliados foram:

Grupo 1 - Sistema de confinamento em gaiolas individuais na fase de gestação e lactação.

Grupo 2 - Sistema de criação em piquete na fase de gestação e baias individuais na fase de lactação.

No primeiro grupo, as marrãs foram alojadas em gaiolas de gestação de 2,2 × $0,6 \times 1,1 \mathrm{~m}$ (comprimento $\mathrm{x}$ largura $\mathrm{x}$ altura), dotada de um comedouro e bebedouro automático do tipo concha e nas gaiolas de lactação de 2,06 x 1,80 em ferro redondo 3/4, 5/8, base de sustentação da fêmea em ferro $3 / 8$ e leitão em $5 / 16$ com base de sustentação em viga fibra de vidro com escamoteador de madeira, incluso bebedouro chupeta para fêmea e leitão, com cocho para fêmea e leitão. As marrãs do segundo grupo foram alojadas na fase de gestação em piquetes coletivos numa área de $2400 \mathrm{~m}^{2}\left(600 \mathrm{~m}^{2}\right.$ por marrã) e na maternidade foram alojadas em baias individuais, de alvenaria, dotadas de cocho manual e bebedouro tipo chupeta, com piso de concreto e telhas de barro tipo francês, dispondo de $14 \mathrm{~m}^{2}$ por fêmea, com acesso à área descoberta. As baias eram dotadas de escamoteador construído em alvenaria, com lâmpadas incandescentes como forma de aquecimento para os leitões.

As variações do ambiente térmico foram monitoradas diariamente por meio de termômetro de máxima e mínima, termômetro de bulbo seco e úmido e termômetro de globo negro localizados dentro do galpão em uma baia separada, dispostos a $60 \mathrm{~cm}$ de altura do piso. Os dados de temperatura, umidade e radiação obtidos foram posteriormente convertidos em Índice de Temperatura de Globo Negro e Umidade (ITGU). Tal índice é baseado na seguinte equação:

$\mathrm{ITGU}=\mathrm{Tg}+0,36 \mathrm{To}+41,5$, sendo que:

$\operatorname{Tgn}=$ temperatura de globo negro;

To $=$ temperatura de ponto de orvalho.

Enquanto To pode ser obtida pela seguinte equação:

To $=\mathrm{T}-[(100-\mathrm{UR}) / 5]$, em que:

$\mathrm{T}=$ temperatura média;

$\mathrm{UR}=$ umidade relativa do ar. 
A ração utilizada foi a base de milho, trigo e farelo de soja, para atender às exigências nutricionais dos animais de acordo com Rostagno et al. (2017). As rações fornecidas para as marrãs foram divididas em três fases: primeira (confirmação de gestação até 75 dias de gestação), segunda (75 dias de gestação até o parto) e terceira (lactação).

Antes do início do experimento as marrãs receberam alimentação ad libitum. As fêmeas foram inseminadas a partir do terceiro cio, utilizando o sêmen do mesmo reprodutor. As rações foram fornecidas duas vezes ao dia, totalizando aproximadamente $2,7 \mathrm{~kg}$ de ração por dia conforme recomendação do manual da linhagem. Os animais tiveram livre acesso aos bebedouros durante todo o período experimental.

Após inseminação, a fêmea foi direcionada ao seu grupo, onde foram coletados dados de comportamento de cada animal, durante a fase de gestação. As matrizes foram devidamente identificadas com uma numeração no dorso, variando de 1 a 4 para os seus respectivos sistemas de alojamento, o que possibilitou a coleta comportamental individual de cada animal.

As observações comportamentais foram realizadas de forma direta, com determinação instantânea. As coletas comportamentais foram realizadas em cinco dias pré-determinados $(1,21,41,61$ e 81 dias) durante a fase de gestação de todas as marrãs, e foram determinados três horários para observação: das 8:00 ás 10:00, das 12:00 ás 14:00 e das 16:00 às 18:00, totalizando seis horas de observação para cada dia selecionado. As coletas comportamentais foram feitas em intervalos de um minuto totalizando 3600 observações por marrã.

Os comportamentos avaliados foram: Inativo (I) - Deitar totalmente (ventral e/ou lateralmente) com os olhos fechados e sem nenhuma atividade oral e Alerta (A) - Parar em pé, deitar ou sentar com os olhos abertos.

As marrãs foram pesadas antes da inseminação para a determinação do peso médio. Aos 100 dias de gestação, os animais foram colocados em baias de lactação, onde permaneceram até o desmame. Para a determinação dos índices zootécnicos, o parto das fêmeas foi acompanhado a fim de saber a gestação em dias (G); o tamanho da leitegada (TL); o número de nascidos vivos (NV), natimortos (NT) e mumificados (MU). Após o fim do parto, foi feita a pesagem dos leitões NV para a determinação do peso vivo (PV) desses animais no primeiro dia de vida. Os leitões foram pesados aos 7, 14, 21 e 28 dias de idade para a determinação do peso ao desmame (PD) e do ganho médio diário (GMD) dos leitões durante todo o período. Foi calculada a taxa de mortalidade $(\%)$ dos animais nascidos vivos até o desmame de cada grupo.

Os dados obtidos a partir do comportamento e desempenho dos animais foram submetidos ao teste de Cramer-von Mises, para verificação da normalidade da distribuição. Em seguida os dados foram submetidos à análise de variância pelo teste $\mathrm{F}$ a $5 \%$ de probabilidade, por meio do programa $\mathrm{R}$ version 3.3.0. Verificando-se significância, utilizou-se o teste de Tukey a 5\% de probabilidade. Para apresentação dos resultados de comportamento, as tabelas foram confeccionadas convertendo-se os valores obtidos em escala percentual.

\section{Resultados e discussão}

A temperatura média ao longo do experimento foi de $18,67 \pm 1,33^{\circ} \mathrm{C}$ com ITGU de $72,5 \pm 2,65$. Os resultados das atividades comportamentais das marrãs na fase de gestação são apresentados na Tabela 1.

As marrãs na fase de gestação alojadas em gaiolas de confinamento, permaneceram na maior $(\mathrm{P}<$ 0.001) parte do tempo inativas nos três horários e menor tempo em alerta entre os horários de 08:00 10:00 e 12:00 - 14:00, comparado as marrãs criadas em piquete. Isso demonstra que as fêmeas alojadas em piquetes exploraram mais o ambiente, principalmente nos horários da manhã e no início da tarde, quando comparado com as marrãs alojadas em gaiolas de gestação. Verificou-se que os animais alojados em piquetes permaneceram $87,3 \%$ em alerta, acima do tempo comparado com as fêmeas alojadas em gaiolas individuais.

Uma possível explicação para os resultados de comportamento se deve as marrãs alojadas em piquetes estarem soltas e apresentarem maior interação entre elas, diferente do que acontece com as marrãs confinadas em gaiolas. Este fato foi observado nos três horários de coleta. A avaliação do bem- 
estar animal na exploração zootécnica pode envolver aspectos ligados às instalações, manejo, ambiente e, principalmente, à resposta do animal ao meio no dia-a-dia na unidade de produção, buscando produtos éticos e de qualidade, o que torna necessário estabelecer critérios que avaliem o bem-estar dos suínos em seus sistemas de criação (Baptista et al., 2011).

Tabela 1. Média das atividades comportamentais de marrãs submetidas a dois modelos de criação na fase de gestação.

\begin{tabular}{lcccc}
\hline Comportamentos $^{1}$ & Gaiola & Piquete & P-valor & CV, \% \\
\hline${\text { Primeiro } \text { horário }^{3}}^{3}$ & & & & \\
\hline Inativo & 93,93 & 46,87 & 0,001 & 17,13 \\
Alerta & 20,47 & 38,87 & 0,01 & 24,05 \\
\hline Segundo horário & & & & \\
\hline Inativo & 102,4 & 39,2 & 0,001 & 28,18 \\
Alerta & 10,6 & 47,1 & 0,001 & 38,93 \\
\hline Terceiro horário & & & & 38,51 \\
\hline Inativo & & 33,4 & 0,001 & 43,39 \\
Alerta & 63,3 & 52,4 & $\mathrm{NS}^{2}$ &
\end{tabular}

${ }^{1}$ Dados diferem estatisticamente pelo teste $\mathrm{F}$ a $1 \%$ de probabilidade $;{ }^{2} \mathrm{NS}$ - Não significativo.

A inatividade dos animais alojados nas gaiolas de gestação foi aproximadamente $54,0 \%$ do tempo total de observação (Tabela 1), superior $(\mathrm{P}<0,01)$ aos animais que ficaram alojadas em piquetes. Esses resultados não corroboram com os apresentados por Silva et al. (2008) e Ramos et al. (2009), que não obtiveram efeito com relação a esses comportamentos entre os sistemas de baias individuais e piquetes coletivos. Gonçalves et al. (2014) compararam o comportamento de fêmeas suínas alojadas em baias individuais com aquelas alojadas em baias coletivas e encontraram uma maior inatividade em fêmeas alojadas em piquetes coletivos, não corroborando com nosso estudo.

O comportamento de alerta observado nos animais caracteriza, desta forma, a maior possibilidade de exploração do ambiente, o que é evidenciado pelo ato de fuçar o solo realizando movimentos circulares, promovendo um comportamento característico da espécie. Esses resultados estão de acordo com os encontrados por Silva et al. (2008) e Ramos et al. (2009), mas diferem dos encontrados por Gonçalves et al. (2014), que não encontraram diferença nesse comportamento nos tipos de alojamentos estudados. Comparado com outros sistemas, o sistema de piquetes contendo pastagens oferecem aos suínos uma oportunidade da realização de atividade física, o que resulta em melhor bem-estar animal (Blumetto Velazco et al., 2013; Olczak et al., 2015).

A Tabela 2 mostra a comparação entre as médias observadas nos grupos sobre a resposta dos seguintes índices zootécnicos: peso à inseminação, período de gestação em dias, tamanho da leitegada, número de leitões nascidos vivos e natimortos.

Tabela 2. Média dos índices de desempenho de marrãs mantidas em diferentes sistemas de alojamentos durante a gestação.

\begin{tabular}{lcccc}
\hline Variáveis & Gaiola & Piquete & P < valor & $\mathrm{CV}, \%$ \\
\hline Peso a inseminação (kg) & 122,6 & 119,2 & $\mathrm{NS}^{1}$ & 3,16 \\
Gestação (dias) & 116,0 & 116,3 & $\mathrm{NS}$ & 0,79 \\
Tamanho de leitegada $\left(\mathrm{n}^{\circ}\right)$ & 8 & 17,5 & 0,01 & 3,92 \\
Nascidos vivos $\left(\mathrm{n}^{\circ}\right)$ & 7 & 14 & 0,04 & 9,52 \\
Natimortos $\left(\mathrm{n}^{\circ}\right)$ & 2 & 1 & $\mathrm{NS}$ & 94,28 \\
\hline
\end{tabular}

${ }^{1} \mathrm{NS}$ - Não significativo

A gestação da fêmea suína tem duração em torno de $114 \pm 3$ dias, com pequenas variações entre granjas ou raças/linhagens. Não houve diferença $(P>0,05)$ na duração do período de gestação, para os dois grupos avaliados. Essa duração encontra-se dentro dos limites esperados, não tendo sido detectadas patologias que pudessem prolongar o período de gestação, para ambos os tratamentos.

No sistema de piquetes houve maior número de nascidos vivos e maior tamanho de leitegada $(\mathrm{P}<$ 0,05). O número de leitões nascidos vivos apresentou variação entre os sistemas de alojamento, onde praticamente dobrou o número de leitões nascidos vivos no sistema de criação em piquete/baia, 
comparado com as fêmeas criadas em gaiolas. Esses resultados podem impactar no número de leitões desmamados por porca/ano. Isso pode ser explicado pelo aumento do comportamento exploratório das fêmeas alojadas em piquetes que pode ter reduzido o estresse relacionado ao confinamento em gaiolas e, consequentemente, influenciando no desempenho reprodutivo das porcas.

Os principais fatores que influenciam diretamente o tamanho da leitegada são ordem de parto, idade da matriz na concepção, número de coberturas por cio, época do ano (temperatura e fotoperíodo), nutrição e sanidade (Silva et al., 2008). Admitindo-se que esses fatores de interferência foram controlados, possivelmente essa variação pode ter sido atribuída ao estresse crônico causado pela falta de espaço às matrizes nas gaiolas de gestação.

Os índices de desempenho dos leitões são apresentados na Tabela 3. Não houve efeito $(\mathrm{P}>0,05)$ entre os sistemas de alojamento em função dos índices de desempenho dos leitões avaliados.

Tabela 3. Média dos índices de desempenho (kg) de leitões nascidos de fêmeas mantidas em diferentes sistemas de alojamentos durante a gestação

\begin{tabular}{|c|c|c|c|c|}
\hline Variáveis $^{1}$ & Gaiola & Piquete & P-valor & $\mathrm{CV}(\%)$ \\
\hline Peso ao nascimento & 1,4 & 1,3 & $\mathrm{NS}^{2}$ & 19,07 \\
\hline Peso aos 7 dias de idade & 2,8 & 2,3 & NS & 23,02 \\
\hline Peso aos 14 dias de idade & 5,1 & 3,6 & NS & 23,75 \\
\hline Peso aos 21 dias de idade & 6,9 & 4,6 & NS & 23,21 \\
\hline Peso aos 28 dias de idade & 8,8 & 6,1 & NS & 21,00 \\
\hline Ganho de peso diário 0-7 dias de idade & 0,2 & 0,1 & NS & 32,60 \\
\hline Ganho de peso diário $0-14$ dias de idade & 0,3 & 0,2 & NS & 28,09 \\
\hline Ganho de peso diário 0-21 dias de idade & 0,3 & 0,2 & NS & 29,47 \\
\hline Ganho de peso diário $0-28$ dias de idade & 0,3 & 0,2 & NS & 22,40 \\
\hline
\end{tabular}

${ }^{1}$ Dados diferem estatisticamente pelo teste $\mathrm{F}$ a $5 \%$ de probabilidade. ${ }^{2} \mathrm{NS}$ - Não significativo.

Com relação ao peso durante o nascimento, ficou constatado que as leitegadas menores apresentaram maior peso, resultado que corrobora com os encontrados por Theil et al. (2010), que avaliaram o desempenho reprodutivo de marrãs da raça Moura em diferentes sistemas de alojamento na fase de gestação. Em uma avaliação anterior, realizada no mesmo rebanho por Mello et al. (2006), considerando-se fêmeas de diferentes ordens de parição e diferentes graus de sangue Moura $(1 / 2 ; 1 / 4$ ou $1 / 8$ de sangue Moura), relataram maiores pesos ao nascimento $(1,54 \mathrm{~kg})$; porém, menores pesos ao desmame $(7,83 \mathrm{~kg})$.

Para que um leitão tenha boa possibilidade de desenvolvimento, seu peso mínimo deve ser igual ou superior a $1,2 \mathrm{~kg}$, ou de 0,7 a $1,2 \mathrm{~kg}$, no caso de transferência cruzada de leitões em instalações em que se trabalha com grupos de fêmeas (Wolf et al., 2011). Em nosso trabalho, não houve a necessidade da transferência de animais entre as fêmeas de cada um dos grupos estudados. Dessa maneira, a leitegada foi constituída por leitões com peso uniforme, o que permitiu melhor desenvolvimento. O peso médio, no nascimento dos leitões oriundos das fêmeas alojadas em gaiolas individuais de gestação, foi de 1,4 $\mathrm{kg}$, e das matrizes em piquetes, de 1,3 kg. Em ambos os métodos de alojamento, o ganho de peso diário atingiu valores mínimos exigidos, aproximadamente $0,19 \mathrm{~kg} / \mathrm{dia}$. Isso garantiu um desmame aos 28 dias de $8,8 \mathrm{~kg}$ para leitões nascidos de matrizes submetidas às gaiolas de gestação e maternidade e de $6,1 \mathrm{~kg}$ para fêmeas submetidas ao piquete na fase de gestação e em baias individuais na fase de maternidade.

As matrizes submetidas ao piquete na fase de gestação tiveram condições melhores de bem-estar que podem ter proporcionado melhor desenvolvimento aos leitões, comparado com as fêmeas alojadas nas gaiolas. Apesar dos índices serem superiores aos valores mínimos sugeridos para peso dos leitões no nascimento e ao desmame, eles não ultrapassaram os índices das fêmeas alojadas em gaiolas. Esses acréscimos nos índices embora irrisórios apontam reflexo direto no cômputo geral da propriedade, garantido o sucesso econômico do negócio, aumentando os lucros e viabilizando as alterações no sistema de alojamento. A avaliação do bem-estar animal na exploração zootécnica pode envolver aspectos ligados às instalações, ao manejo, ao ambiente e, principalmente, à resposta do animal ao meio no diaa-dia na unidade de produção, buscando interpretar a linguagem animal, ou seja, a resposta comportamental frente aos regimes de criação (Silva et al., 2008; MAPA, 2016). 
O baixo número de repetições também pode ter relação com o resultado final sobre os parâmetros avaliados, levando em consideração as altas taxas de coeficiente de variação $(\mathrm{CV})$ nesse experimento (Tabelas 1, 2 e 3). Quando se trata de matrizes em gestação, se faz necessário o uso de um maior número de repetições de modo a aumentar o grau de liberdade do resíduo, diminuindo assim as margens de erro experimental. Esses resultados foram semelhantes aos encontrados por Silva Júnior et al. (2015), que trabalharam com marrãs em gestação.

Considerando que o período total de gestação da porca é relativamente longo e há uma grande quantidade de fatores que cada sistema de alojamento (Fase de gestação e maternidade) pode influenciar o desempenho da leitegada, mais trabalhos científicos são necessários para uma determinação mais precisa do uso ou não de gaiolas na gestação.

\section{Conclusão}

O sistema de piquetes na gestação reduziu a inatividade das marrãs proporcionando um melhor bemestar, que foi suficiente para melhorar os índices de número de nascidos vivos e tamanho de leitegada, mostrando que diferentes sistemas de alojamento podem apresentar diferentes índices de desempenho e repostas comportamentais.

\section{Agradecimentos}

O presente trabalho foi realizado com apoio da Universidade Estadual do Norte Fluminense Darcy Ribeiro, com a concessão da bolsa de Pós-doutorado ao primeiro autor.

\section{Referências}

ANUALPEC. (2020). Anuário da Pecuária Brasileira (20th ed., Vol. 1). Instituto FNP.

FAPRI. (2020). Food and Agricultural Policy Research Institute (W. A. O. Database (ed.)). Food and Agricultural Policy Research Institute; Iowa State University and University of Missouri-

Columbia. http://www.fapri.iastate.edu/tools/outlook.aspx.

Baptista, R. I. A. A., Bertani, G. R. \& Barbosa, C. N. (2011). Indicadores do bem-estar em suínos. Ciencia Rural, 41, 1823-1830. DOI: https://doi.org/10.1590/s0103-84782011005000133

Blumetto Velazco, O. R., Calvet Sanz, S., Estellés Barber, F. \& Villagrá García, A. (2013). Comparison of extensive and intensive pig production systems in Uruguay in terms of ethologic, physiologic and meat quality parameters. Revista Brasileira de Zootecnia, 42, 521-529. DOI: https://doi.org/10.1590/s1516-35982013000700009.

Budiño, F. E. L., Vieira, R. F.N., Mello, S. P. \& Keila M. R. (2014). Duarte1 Behavior and performance of sows fed different levels of fiber and reared in individual cages or collective pens. Anais da Academia Brasileira de Ciências, 86(4): DOI: http://dx.doi.org/10.1590/0001-3765201420140301

Gonçalves, T. M., Bastos, R., Soares, R. T. N. \& Torres, D. S. (2014). Indicadores comportamentais na avaliação do bem-estar de matrizes de suínos alojadas em baias individuais e piquetes coletivos. Jornal Brasileiro de Ciência Animal, 7, 523-540.

Kongsted, H. \& Sørensen, J. T. (2017). Lesions found at routine meat inspection on finishing pigs are associated with production system. The Veterinary Journal, 223, 21-26. DOI: https://doi.org/10.1016/j.tvjl.2017.04.016.

Köppen, W., \& Geiger, R. (1928). Klimate der Erde. Gotha: Verlag Justus Perthes. Wall-Map $150 \mathrm{~cm} \times 200 \mathrm{~cm}$.

MAPA. (2016) Disponível em: <http://www.agricultura.gov.br/desenvolvimento9sustentavel/producao-integrada-cadeia-pecuaria/boas-praticas-agropecuarias >. Acesso em: 15 set. 2016.

Mello, G., Carvalho, M., Chiquitelli Neto, M., Laurentiz, A. C., Bastos, J. F. P. (2006). Levantamento da qualidade dos leitões da raça moura produzidos na UNESP de Ilha Solteira. In II Semana de Zootecnia da FE-UNESP-Ilha Solteira, CD-Rom.

Nazareno, A. C., Silva, I. J. O., Nunes, M. L. A., Castro, A. C., Késia, O. S., Miranda, K. O. S. \& Trabachini, A. (2012). Caracterização bioclimática de sistemas ao ar livre e confinado para a criação 
de matrizes suínas gestantes. Revista Brasileira de Engenharia Agrícola e Ambiental, 16, 314-319. DOI: https://doi.org/10.1590/s1415-43662012000300013

Olczak, K., Nowicki, J. \& Klocek, C. (2015). Pig behaviour in relation to weather conditions- A review. Annals of Animal Science, 15, 601-610.

Oliva, A., Tama, B., Elias, D., Barboza, K., Pereira, D.A. \& Oliveira, L. G. (2014). Aspectos de bemestar relacionados a matrizes suínas alojadas em celas individuais. Relato de caso. Revista Brasileira de Higiene e Sanidade Animal, 8, 89-104.

Ramos, A. M., Santos, L. A. R. \& Fontes, L. T. G. (2009). Normais climatológicas do Brasil. Brasília, DF, INMET.

Rostagno, H. S.; Albino, L. F. T.; Hannas, M. I.; Donzele, J. L.; Sakomura, N. K.; Perazzo, F. G.; Saraiva, A.; Abreu, M. L. T.; Rodrigues, P. B.; Oliveira, R. F.; Barreto, S. L. T. \& Brito, C. O. (2017). Tabelas Brasileiras para Aves e Suínos (4th ed). Viçosa, UFV, Departamento de Zootecnia, Brasil.

R VERSION 3.3.0 (2016) -- "Supposedly Educational" Copyright (C). The R Foundation for Statistical Computing Platform: i386-w64-mingw32/i386 (32-bit).

Silva, I. J. O., Pandorfi, H. \& Piedade, S. M. S. (2008). Influência do sistema de alojamento no comportamento e bem-estar de matrizes suínas em gestação. Revista Brasileira de Zootecnia, 37, 1319-1329. DOI: https://doi.org/10.1590/s1516-35982008000700025

Silva Júnior, E. T., Soares, R. T. R. N., Molino, J. P., Oliveira, R. F., Bonaparte, T. P. \& Silva, L. F. A. (2015). Lisina digestível para marrãs no terço final da gestação. Archives of Veterinary Science, 20, 01-09. DOI: https://doi.org/10.5380/avs.v20i4.41668

Sousa, M. S., Tinoco, I. F. F., Inoue, K. R. A., Pereira, A. K. \& Sousa, F. C. (2012). Comportamento e Bem-estar de porcas gestantes. In: Marcos Oliveira de Paula; Ilda de Fátima Ferreira Tinôco; Cecília de Fátima Souza; Fernando da Costa Baêta. (Org.). Construções Rurais e Ambientes Protegidos: Inovações Tecnológicas e Produção Animal em Condições de Clima Quente. 1ed.Viçosa: Editora UFV, 2012, p. 225-229.

Theil, P., Akechi, B. V., Rodrigues, D. C., Andrade, M. F., Tedeschi, L. \& Filardi, R. S. (2010). Desempenho de marrãs da raça Moura mantidas em diferentes sistemas de alojamento durante a fase de gestação. Faculdade de Engenharia - Campus de Ilha Solteira-UNESP, 2010.

Veloni, M. L., Prado, P. L., Arssuffi, B. M., Ballester, M. C. M., Oliveira, M. G., Abreu, P. B. \& Oliveira, L. G. (2013). Bem-estar animal aplicado nas criações de suínos e suas implicações na saúde dos rebanhos. Revista Cientifica Eletrônica de Medicina Veterinária, 11(21) 1-21.

Wolf, J. B., Leamy, L. J., Roseman, C. C. \& Cheverud, J. M. (2011). Disentangling prenatal and postnatal maternal genetic effects reveals persistent prenatal effects on offspring growth in mice. Genetics, 189, 1069-1082. DOI: https://doi.org/10.1534/genetics.111.130591

Histórico do artigo:

Recebido: 3 de julho de 2020 .

Aprovado: 5 de agosto de 2020 .

Disponível online: 9 de dezembro de 2020 Licenciamento: Este artigo é publicado na modalidade Acesso Aberto sob a licença Creative Commons
Atribuição 4.0 (CC-BY 4.0), a qual permite uso irrestrito, distribuição, reprodução em qualquer meio,
desde que o autor e a fonte sejam devidamente creditados. 